\title{
Promoção da saúde do homem: reflexão docente
}

\section{Promoting man's health: teacher reflection}

DOI: $10.46919 / \operatorname{archv1n5-003~}$

Recebimento dos originais: 10/07/2020

Aceitação para publicação: 30/08/2020

\section{Maria Nauside Pessoa da Silva}

Graduada em Enfermagem; Teóloga

Mestra em Saúde da Família (UNINOVAFAPI)

Instituição: Faculdade Uninassau - Aliança: Rua Dr. Otto Tito, 278-306 - Redenção Teresina /Piauí/Brasil

Instituição: Faculdade de Educação do Piauí - FAEPI. Rua Treze de Maio 2660 - Teresina - PI, (PIAUÍ/BRASIL)

Instituição: Faculdade Tecnológica de Teresina - CET. Rua Rio Grande do Norte, 790 - Pirajá, Teresina PI, (PIAUÍ/BRASIL)

E-mail: nauside@yahoo.com.br

\section{Antonio Marçal de Sousa Val}

Graduado em medicina pela Universidade Federal do Maranhão

Mestre em Saúde da Família (UNINOVAFAPI)

Instituição: Universidade Federal do Piauí -UFPI. Av. São Sebastião, no 2819 - Nossa Sra. de Fátima,

Parnaíba - PI (PIAUÍ/BRASIL)

E-mail: amasoval@hotmail.com

\section{Caroline Murad Abdalla}

Graduada em Fisioterapia

\section{Mestra em Saúde da Família (UNINOVAFAPI)}

Instituição: Centro Universitário - UNDB -Av. Colares Moreira, 443 - Jardim Renascença, São Luís MA (MARANHÃO/BRASIL)

E-mail: carolineabdalla@hotmail.com

\section{Gladys Carvalho de Araújo Alencar}

Graduada em Medicina.

Mestra em Saúde da Família (UNINOVAFAPI)

Instituição: Universidade Estadual do Piauí - UESPI. Rua Olavo Bilac, 2335 - Centro (Sul), Teresina PI. (PIAUÍ/BRASIL)

E-mail: gladysalencar@yahoo.com.br

\section{Lorena Rocha Batista Carvalho}

Graduada em Enfermagem

Mestra em Saúde da Família (UNINOVAFAPI)

Instituição: Centro Universitário do Piauí - UNIFAPI. Rua Gov. Joca Píres, 1000 - Fátima, Teresina - PI (PIAUÍ/BRASIL)

E-mail: lorenarbcarvalho@gmail.com 


\section{Lennara de Siqueira Coêlho}

Graduada em Enfermagem

Mestra em Saúde da Família (UNINOVAFAPI)

Instituição: Centro Universitário do Piauí - UNIFAPI. Rua Gov. Joca Píres, 1000 - Fátima, Teresina - PI (PIAUÍ/BRASIL)

E-mail: lennara.coelho@hotmail.com

\section{Lília Maria Monteiro de Oliveira e Silva}

Graduada em Nutrição

Mestra em Saúde da Família (UNINOVAFAPI)

Instituição: Faculdade Estácio de Sá Unidade Teresina. Av. dos Expedicionários, 790 - São João,

Teresina - PI. (PIAUÍ/BRASIL)

E-mail: oliveira.lilia@estacio.br

\section{Marcelo de Moura Carvalho}

Graduado em Enfermagem

Doutor em Políticas de Saúde - Universidade Federal do Piauí - UFPI

Instituição: Fundação Municipal de Saúde - FMS. Rua Gov. Raimundo Artur Vasconcelos, 3015 -

Primavera, Teresina - PI. (PIAUÍ/BRASIL)

E-mail: Marcelo.mcarvalho@yahoo.com.br

\section{Teresa Marly Teles de Carvalho Melo Cirurgiã Dentista}

Mestra em Saúde da Família (UNINOVAFAPI)

Doutora em Engenharia Biomédica - UNIVAPI

Instituição: Instituto Federal do Piauí - IFPI. Rua Álvaro Mendes, 94 - Centro (Sul), Teresina - PI (PIAUÍ/BRASIL)

E-mail: hyramelo@yahoo.com.br

\section{RESUMO}

O estudo apresenta um relato de experiência realizado por Docentes e Acadêmicos de Enfermagem, tendo como objetivos: Desenvolver ações sócias - educativas que desperte nos funcionários a compreensão do cuidado à saúde; Promover ações primordiais à manutenção da saúde e de bem - estar; Propiciar conhecimento acerca das diversas patologias, causas, consequências e prevenção. METODOLOGIA: As atividades foram realizadas em uma Empresa de Transportes. A realização das ações ocorreu por meio de abordagem de diversas temáticas tendo como escopo a Política Nacional de Atenção Integral à Saúde do Homem. RESULTADOS E DISCUSSÃO: Setenta funcionários participaram das atividades todos do gênero masculino, com idade entre 20 e 74 anos. Para realização das atividades foram desenvolvidas diversas ações como: palestras, oficinas, roda de conversa e distribuição de folhetos educativos sobre as temáticas abordadas, assim como a realização de aferição de pressão arterial e glicemia capilar. Concluiuse que às ações sócias - educativas direcionadas para a promoção da saúde e prevenção de doenças no público masculino foi de importante significado, tanto pela interação com os funcionários da empresa de transportes, quanto pelas atividades realizadas envolvendo as educativas e a prestação de serviços de saúde.

Palavras-chave: Saúde do homem, Enfermagem, Promoção da saúde, Prevenção de doenças.

\section{ABSTRACT}

The study presents an experience report carried out by Nursing Professors and Academics, with the following objectives: Developing social - educational actions that awaken the employees' understanding 
of health care; Promote primary actions for the maintenance of health and well - being; Provide knowledge about the various pathologies, causes, consequences and prevention. METHODOLOGY: The activities were carried out in a Transport Company. The actions were carried out through the approach of several themes with the scope of the National Politics for Integral Attention to Men's Health. RESULTS AND DISCUSSION: Seventy employees participated in activities, all male, aged between 20 and 74 years. In order to carry out the activities, several actions were developed, such as: lectures, workshops, conversation circle and distribution of educational leaflets on the topics covered, as well as the measurement of blood pressure and capillary blood glucose. It was concluded that the social - educational actions directed to health promotion and disease prevention in the male public were of significant significance, both for the interaction with the employees of the transport company, as well as for the activities carried out involving the educative and the provision of services. of health.

Keywords: Man's health, Nursing, Health promotion, Prevention of diseases.

\section{INTRODUÇÃO}

O Ministério da Saúde, nos vinte anos do SUS, apresenta a Política Nacional de Atenção Integral à Saúde do Homem, tendo como principais objetivos promover ações de saúde que contribuam para a compreensão da realidade masculina nos seus diversos contextos socioculturais. O Ministério da Saúde vem cumprir seu papel ao formular a política norteadora das ações de atenção integral à saúde do homem, estimulando o reconhecimento que a saúde é um direito social e de cidadania de todos, orientar as ações e serviços de saúde para a população masculina, com integralidade, equidade e humanização da atenção. Diretrizes, entender a saúde do homem como um conjunto de ações de promoção, prevenção, assistência e recuperação da saúde, em foco a educação, como promotora de novas formas de pensar e agir (BRASIL, 2008). A subjetividade que na Nova Promoção à Saúde induz é baseada na imagem de um indivíduo racional, civilizado, e consciente de sua saúde e bem-estar. Proposições que inspiraram o Ministério da Saúde na adoção de medidas para estimular a população masculina a cuidar da saúde, não só no estado curativo mas sobretudo em caráter preventivo (BRASIL, 2009).

\section{OBJETIVOS}

Desenvolver ações sócias - educativas que desperte nos funcionários a compreensão do cuidado à saúde; Promover ações primordiais à manutenção da saúde e de bem - estar; Propiciar conhecimento acerca das diversas patologias, causas, consequências e prevenção.

A Política Nacional de Saúde do Homem é desenvolvida a partir de cinco (05) eixos temáticos: 1. Acesso e Acolhimento: objetiva reorganizar as ações de saúde, através de uma proposta inclusiva, na qual os homens considerem os serviços de saúde também como espaços masculinos e, por sua vez, os serviços reconheçam os homens como sujeitos que necessitam de cuidados.

2. Saúde Sexual e Saúde Reprodutiva: busca sensibilizar gestores(as), profissionais de saúde e a população em geral para reconhecer os homens como sujeitos de direitos sexuais e reprodutivos. 
3. Paternidade e Cuidado: objetiva sensibilizar gestores(as), profissionais de saúde e a população em geral sobre os benefícios do envolvimento ativo dos homens em todas as fases da gestação e nas ações de cuidado com seus(uas) filhos(as), destacando como esta participação pode trazer saúde, bem-estar e fortalecimento de vínculos saudáveis entre crianças, homens e suas (eus) parceiras(os).

4. Doenças prevalentes na população masculina: busca fortalecer a assistência básica no cuidado à saúde dos homens, facilitando e garantindo o acesso e a qualidade da atenção necessária ao enfrentamento dos fatores de risco das doenças e dos agravos à saúde.

5. Prevenção de Violências e Acidentes: visa propor e/ou desenvolver ações que chamem atenção para a grave e contundente relação entre a população masculina e as violências (em especial a violência urbana) e acidentes, sensibilizando a população em geral e os profissionais de saúde sobre o tema (MINISTÉRIO DA SAÚDE. 2020).

\section{METODOLOGIA}

As atividades foram realizadas em uma Empresa de Transportes. A realização das ações ocorreu por meio de abordagem de diversas temáticas, como: infecções sexualmente transmissíveis, câncer de pênis e de próstata, obesidade, doenças cardiovasculares e diabetes, como prevenir e detectar precocemente. A atividade contou ainda com a realização de aferição de pressão arterial e glicemia capilar, cujas ações contou com a participação de Acadêmicos do curso de Enfermagem de uma Instituição de Ensino Superior, supervisionados pela Professora/Enfermeira.

\section{RESULTADOS E DISCUSSÃO}

Participaram das atividades 70 funcionários, todos do gênero masculino, com idade entre 20 e 74 anos. Durante as atividades foram realizadas palestras, oficinas, roda de conversa e distribuição de folhetos educativos sobre as temáticas abordadas, realização de aferição de pressão arterial e glicemia capilar.

\section{CONCLUSÃo}

Observou-se que às ações sócias - educativas direcionadas para a promoção da saúde e prevenção de doenças evidenciou plena aceitação do público alvo e proporcionou a valorosa oportunidade dos discentes e docentes aproximação e interação entre teoria/prática. Recomenda-se o desenvolvimento de outras experiências exitosas envolvendo atividades educativas na promoção da saúde e prevenção de doenças. 


\section{REFERÊNCIAS}

BRASIL, Caderno de Atenção Básica nº 16. DIABETES MELLITUS, 2006.

BRASIL, Política Nacional de Atenção Integral à Saúde do Homem, 2008.

BRASIL, Ministério da Saúde, 2009.

FRÁGUAS, Renério et al. Depressão e diabetes mellitus. Rev. Psiquiatria Clínica, São Paulo, v. 36, supl. 3, 2009.

FERREIRA, Joel Saraiva; AYDOS, Ricardo Dutra. Prevalência de Hipertensão Arterial em crianças e adolescentes obesos. Rev. Ciênc. Saúde Coletiva, Rio de Janeiro, v.15, n.1, jan. 2010.

HAMMERSCHMIDT, Karina Silveira de Almeida; LENARDT, Maria Helena. Tecnologia educacional inovadora para o empoderamento junto a idosos com diabetes mellitus. Rev. Texto Contexto Enfermagem, Florianópolis, vl.19, n. 2, abr. /jun., 2010.

MINISTÉRIO DA SAÚDE. Secretaria de Atenção à Saúde. Departamento de Ações Programáticas Estratégicas. Política Nacional de Atenção Integral à Saúde do Homem - Princípios e Diretrizes, Brasília, ago. 2008.

MINISTÉRIO DA SAÚDE. Secretaria de Atenção à Saúde. Saúde do Homem: promoção e prevenção à saúde integral do homem. Política Nacional de Atenção Integral à Saúde do Homem http://saude.gov.br/saude-de-a-z/saude-do-homem - acesso em 26/08/2020

SCHMIDT, Maria Inês et al. Prevalência de diabetes e hipertensão no Brasil baseada em inquérito de morbidade auto-referida, Brasil, 2006. Rev. Saúde Pública, São Paulo, v. .43, supl. 2, nov. 2009. 